\title{
Composición florística de la cuenca del río llo-Moquegua y Lomas de Ilo, Moquegua, Perú
}

\author{
Floristic composition of river Ilo-Moquegua basin and Lomas of Ilo, \\ Moquegua, Peru
}

\author{
Mónica Arakaki y Asunción Cano*
}

Presentado: $26 / 05 / 2003$

Aceptado: 18/06/2003

\section{Resumen}

Se presenta un estudio de la composición y riqueza de la flora vascular en la cuenca del Río lloMoquegua y las Lomas de llo, a lo largo de una gradiente altitudinal que va desde los 0 hasta $4600 \mathrm{~m}$, basado en colectas intensivas, revisión de ejemplares de herbario y consulta bibliográfica. De acuerdo al presente estudio, la flora vascular de la cuenca del río llo-Moquegua y las Lomas de llo está integrada por 63 familias, 233 géneros y 394 especies. Las Magnoliopsida representan el $83 \%$ de las especies y las Liliopsida el $15 \%$. Las familias con mayor número de géneros y especies son Asteraceae (41 géneros y 60 especies), Poaceae (28 y 44), Solanaceae (11 y 32), Fabaceae (17 y 26), Malvaceae (11 y 21), Brassicaceae (10 y 15), Boraginaceae (9 y 15) y Cactaceae (10 y 14). Las formas biológicas dominantes son las hierbas $(72 \%)$, seguidas por los arbustos $(21 \%)$, plantas que pueden ser hierbas o arbustos (5\%), árboles (2\%) y plantas parásitas (menos del 1\%). De las 55 especies endémicas del Perú reportadas en el presente estudio, 10 están restringidas al departamento de Moquegua. Se presenta un total de 176 nuevas adiciones a la flora del departamento.

Palabras clave: flora, Ilo, Moquegua, Perú, lomas, especies endémicas.

\begin{abstract}
A study of the vascular flora composition in the river llo-Moquegua basin and Lomas of llo is presented herein, at elevations that vary between sea level and 4600 meters, based on intensive collections, examination of herbarium specimens and bibliographic research. According to the present study, the vascular flora in the river llo-Moquegua basin and Lomas of llo was found to consist of 63 families, 233 genera and 394 species. Eighty three percent of the species are Magnoliopsida and 15\% belong to the Liliopsida. Families with the largest number of genera and species are Asteraceae (41 genera and 60 species), Poaceae (28 and 44), Solanaceae (11 and 32), Fabaceae (17 and 26), Malvaceae (11 and 21), Brassicaceae (10 and 15), Boraginaceae (9 and 15) and Cactaceae (10 and 14). The dominant biological forms are herbs $(69 \%)$, followed by shrubs $(28 \%)$, climbing plants $(4 \%)$, trees $(2 \%)$ and parasitic plants (less than $1 \%$ ). Fifty five species are endemic to Peru, and 10 are restricted to the department of Moquegua. Total of 176 new additions for the department of Moquegua are presented.
\end{abstract}

Keywords: flora, Moquegua, Ilo, Peru, lomas, endemic species.

\section{Introducción}

La cuenca hidrográfica del Río IloMoquegua se encuentra localizada en el extremo sur occidental del Perú, formando parte de las provincias de Ilo y Mariscal Nieto, en el departamento de Moquegua. Las Lomas de Ilo se hallan a lo largo de la costa,

\footnotetext{
*Museo de Historia Natural, Universidad Nacional Mayor de San Marcos, Av. Arenales 1256, Apartado 14-0434, Lima 14, Perú.
}

eMail: Mónica Arakaki marakaki@lycos.com encontrándose entre las más meridionales del territorio peruano.

De acuerdo con el Plan Director de estrategias del Sistema Nacional de Áreas Protegidas del Perú (INRENA, 1995), la cordillera meridional de los Andes occidentales, que incluye grandes regiones de los departamentos de Arequipa, Moquegua y Tacna, es un área con vacío de información y de prioridad 1 , lo que significa que no se tiene información acerca de su diversidad biológica. Asimismo, re- 
calcan que por razones climáticas y de historia geológica se espera encontrar en estas áreas un alto nivel de endemismo.

La diversidad biológica del departamento de Moquegua ha sido poco estudiada, a pesar de ser conocida la importancia y urgencia de este tipo de trabajos con propósitos de conservación (Ferreyra, 1961; Rodríguez, 1996). Los primeros estudios florísticos fueron los de Ferreyra (1961) en las lomas de Ilo. Posteriormente numerosos investigadores, entre ellos Weberbauer, R. Ferreyra, M. Dillon, C. Vargas, E. Núñez, M. Weigend, O. Pearson, G. Müller, P. Hutchison, K. Gengler y G. Pascaud, realizaron colecciones de flora como parte de proyectos más amplios. Arakaki (1999) realizó un estudio de la flora y vegetación en la cuenca del río Ilo-Moquegua; y más recientemente, Arakaki y Cano (2001) presentaron un panorama de la vegetación de esta cuenca, basándose en toda la información previa.

En este trabajo presentamos los datos sobre composición y riqueza de la flora vascular de la cuenca del río Ilo-Moquegua y de las Lomas de Ilo. Sin embargo, consideramos que se requieren complementar los estudios florísticos con estudios de ecología y biogeografía de esta región, que ha sido y sigue siendo afectada por diversas actividades humanas, así como por fenómenos naturales.

\section{Materiales y métodos}

El área de estudio incluye la franja costera, que básicamente abarca las Lomas de Ilo, y la cuenca del río Ilo-Moquegua. El estudio comprende un transecto altitudinal que se extiende desde su desembocadura en la costa, hasta las montañas y planicies de la puna a los 4600 msnm (Fig. 1).

La colecta del material botánico y los estudios y observaciones en el campo se realizaron entre los años 1995 y 1996. A finales de 1997 se hicieron colectas en las Lomas de Ilo, debido a su desarrollo extraordinario por la presencia del evento «El Niño».
Los métodos empleados han sido los estandarizados para estudios florísticos, que incluyen la colección, herborización e identificación. Para la elaboración de la lista florística se han considerado las colectas depositadas en el Herbario San Marcos (USM) de Lima y Herbario del Museo Field (F) de Chicago.

Con el fin de evaluar la flora de la cuenca del Río Ilo-Moquegua, se comparó numéricamente con la flora de otra región relativamente cercana y que representa una gradiente similar a la nuestra (Sierra de Huaylillas, Volcán Guane Guane, Chile, Kalin et al., 1982). Para el análisis comparativo se ha utilizado el Indice de Similitud de Sorensen (ISS) (GreigSmith, 1964).

\section{Resultados}

La diversidad florística de la cuenca del Río Ilo-Moquegua y las Lomas de Ilo está conformada por 394 especies de plantas vasculares, pertenecientes a 233 géneros y 63 familias.

Los pteridófitos son un grupo taxonómico no muy bien representado, con sólo siete especies $(2 \%)$. Los gnetófitos, con dos especies, que constituyen el $1 \%$ del total de la flora, están representados por un sólo género: Ephedra y dos especies de amplia distribución. Las magnoliópsidas (dicotiledóneas), con 326 especies (83\%), son el grupo mejor representado, siendo herbáceas la mayoría de las especies; y las liliópsidas (monocotiledóneas), con 59 especies, representan el 15\% de la flora. Es importante anotar que 44 de las 59 especies de liliópsidas pertenecen a la familia Poaceae.

Las familias con mayor riqueza de especies son Asteraceae con 60 especies (15\% de las especies), Poaceae con 44 especies (11\%), Solanaceae con 32 especies (8\%), Fabaceae con 26 especies (7\%), Malvaceae con 21 especies $(5 \%)$, Brassicaceae con 15 especies (4\%), Boraginaceae con 15 especies (4\%), Scrophulariaceae con 13 especies (3\%), Cactaceae con 13 especies (3\%) y Euphorbiaceae con nueve especies (2\%). Las 


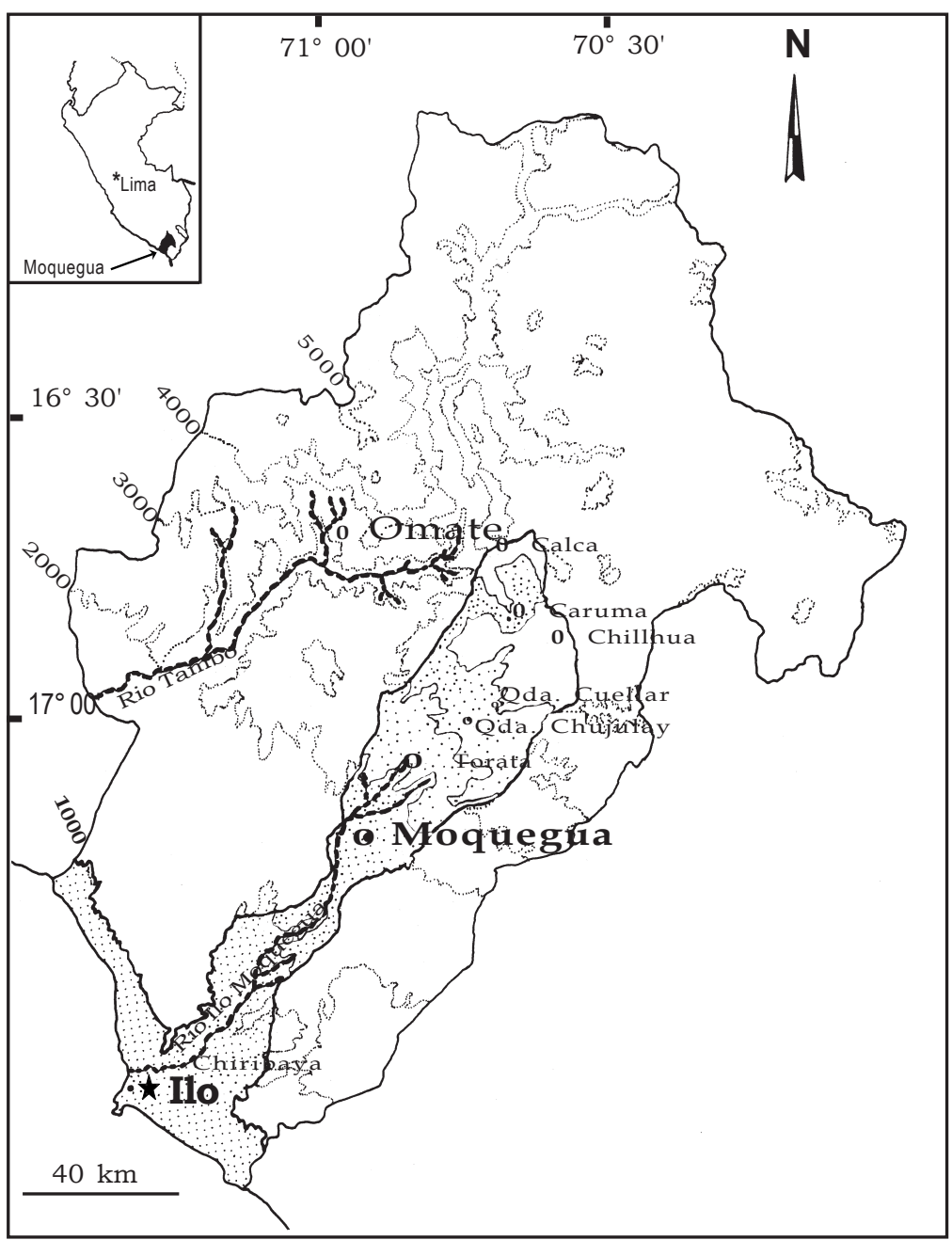

Figura 1. Mapa del Departamento de Moquegua indicando la ubicacion del área de estudio y localidades de muestreo. primeras seis familias hacen el $50 \%$ del total de especies. El número más alto de géneros ha sido encontrado en las familias Asteraceae con 41 géneros, Poaceae (28), Fabaceae (17), Solanaceae (11), Malvaceae (11), Brassicaceae (10), Cactaceae (10), Apiaceae (6), Verbenaceae (5) y Liliaceae (5). Las primeras seis familias constituyen el 50\% del total de géneros.

Las familias con solamente un género son 30 (48\%); 18 familias (29\%) tienen sólo una especie. El número de familias representadas por dos géneros son nueve (15\%) y las representadas por dos especies son 12 (19\%). Tres géneros y tres especies son encontrados en ocho $(13 \%)$ y seis $(10 \%)$ familias respectiva- mente. Un porcentaje considerable de las familias representadas en la zona de estudio posee pocos géneros y especies.

En cuanto a las formas de crecimiento, las especies herbáceas alcanzan los valores más altos, superando por más de dos veces en porcentaje (69\%) al resto de las especies. Le siguen en importancia las formas arbustivas y las plantas trepadoras. Los árboles, a pesar de no tener presencia significativa, son importantes fisionómicamente en el área de estudio. Por su parte, las plantas parásitas están representadas por una sola especie.

El análisis de similitud de la cuenca del río Ilo-Moquegua con la región comprendida entre sierra de Huaylillas y el volcán Guane- 
Tabla 1. Análisis de similitud de la cuenca del río llo-Moquegua y transecto entre Sierra de Huaylillas y volcán Guane-Guane, Chile.

\begin{tabular}{lcccc}
\hline & $\begin{array}{c}\text { Cuenca río } \\
\text { Moquegua, } \\
\text { Perú }\end{array}$ & $\begin{array}{c}\text { Sierra de Huaylillas, } \\
\text { volcán Guane Guane } \\
\text { Chile* }\end{array}$ & Taxa compartidos & $\begin{array}{c}\text { Índice similitud } \\
\text { (ISS) }\end{array}$ \\
\hline Familias & 63 & 47 & 36 & $65,5 \%$ \\
Géneros & 232 & 145 & 87 & $46,2 \%$ \\
Especies & 393 & 275 & 46 & $13,8 \%$ \\
\hline
\end{tabular}

* Kalin et al. (1982)

Guane en Chile, nos muestra una similitud de $65 \%$ a nivel de familias, la similitud a nivel de géneros es de $46 \%$ y a nivel de especies de $14 \%$ (Tabla 1).

\section{Discusión y conclusiones}

Hasta el presente, si bien existían algunos estudios realizados en la cuenca del río IloMoquegua, ellos no revelaban la composición florística de esta región. Ferreyra (1961) reportó 50 especies para las Lomas de Ilo y el presente estudio adiciona 28 especies a las anteriormente reportadas por dicho autor.

Como contribución al conocimiento de la flora de la cuenca del río Ilo-Moquegua y Lomas de Ilo, se reportan 394 especies de plantas vasculares, que representan el 36\% de la flora documentada (Brako \& Zarucchi, 1993) para el suroccidente peruano (departamentos de Ica, Arequipa, Moquegua y Tacna).

Las familias mejor representadas son Asteraceae con 60 especies, Poaceae con 44, Solanaceae con 32, Fabaceae con 26 y Malvaceae con 21. Las Asteraceae y Poaceae constituyen el $26 \%$ del total de especies conocidas para la cuenca estudiada y confirman lo hallado por Gentry (1982) y Young \& Cano (1994), que son las familias dominantes y más ricas en especies en la flora andina.

Se cita por primera vez 176 especies de plantas vasculares para el departamento de Moquegua; y se confirma lo que se sostuvo con anterioridad, que la cuenca del río IloMoquegua ha sido un área muy poco estudiada y con escasas colecciones botánicas, a pesar de ser conocida la urgencia de su estudio para la conservación (Rodríguez, 1996). Cabe mencionar que varias de las especies reportadas en este estudio han sido colectadas una sola vez, son ejemplares tipo y corresponden a las colectas de A. Weberbauer en 1925; otro número importante no se encuentra bien representado en los herbarios, aun siendo especies de amplia distribución.

El valor de la región estudiada no radica en gran medida en el número de especies que alberga, sino en el elevado número de endemismos (Rivas-Martínez \& Tovar, 1983). De las 55 especies reportadas como endémicas para el Perú (13,7\% de la flora), nueve $(2,3 \%)$ están restringidas al departamento de Moquegua (Apéndice 1) y provienen principalmente de la zona altoandina y lomas costeras. Las lomas costeras poseen un alto nivel de endemismo entre las plantas con flores (ver León et al., 2003 para el caso de helechos) y éste alcanza porcentajes mayores en las lomas sureñas (Ferreyra, 1983; Rundell et al., 1991). Varias especies endémicas de la región andina reportadas aquí corresponden a los géneros Malesherbia, Nolana, Calceolaria, Senecio, Drymaria y Hoffmannseggia.

Se ha intentado comparar florísticamente el área de estudio con otros lugares; así, se eligió la región de sierra Huaylillas y volcán 
Guane-Guane (Chile), porque, aunque se sabe que actualmente la parte norte del desierto de Chile (Atacama) separa la costa desértica y los pisos inferiores de las vertientes occidentales peruanas y chilenas, en algún tiempo se ha realizado en esta área un notable intercambio florístico. El clima del suroccidente peruano es más apto para las plantas del norte chileno, que para las que habitan el norte peruano (Weberbauer, 1945). El análisis de similitud de la cuenca del Río Ilo-Moquegua con la región comprendida entre sierra de Huaylillas y el volcán Guane-Guane en Chile muestra una alta similitud a nivel de familias (65\%), debido a que ambas regiones forman parte del mismo sistema montañoso de los Andes del Sur y a que presentan casi las mismas características altitudinales y de vegetación; esta similitud hubiera podido ser mayor de haberse colectado las familias con representantes acuáticos que ellos reportan y que en el presente estudio no se pudieron colectar por razones logísticas. Estas familias (Haloragaceae, Hydrocharitaceae, Lemnaceae, Zannichelliaceaey Potamogetonaceae) poseen un amplio rango de distribución que incluye el área de estudio. Asumiendo la presencia de estas familias la similitud sería del 71\%. La baja similitud específica indicaría el efecto evidente de la barrera desértica de Atacama - que se extiende hasta los $2600 \mathrm{~m}$ de altitud - en la distribución de la flora y fauna andinas en ambos extremos de ella. Las afinidades genéricas relativamente mayores indicarían que las distribuciones a lo largo de toda esta área habrían sido más amplias en el pasado.

\section{Agradecimientos}

Nuestro reconocimiento por el importante apoyo en las diferentes etapas del desarrollo del presente trabajo a Joaquina Albán, Hamilton Beltrán, Elida Carrillo, Emma Cerrate, Magda Chanco, Rosa Choque, Michael Dillon, Ramón Ferreyra, Jaime Filinich, Robin Foster, Karla Gengler, Laura Gilliam, Nancy Hensold, María I. La Torre, Blanca León, Nelly Llerena, Raúl Menaut, Ana Miranda, Carlos Ostolaza, Nancy Refulio,
Juan Revilla, José Roque, Flor Salvador, Laura Torres, Oscar Tovar, Adán Umire, Graciela Vilcapoma, Tatzyana Wachter, Maximilian Weigend y Kenneth Young. Un sincero agradecimiento a los directivos de los herbarios San Marcos del Museo de Historia Natural de Lima (USM) y Museo Field de Chicago (F) por el apoyo en la consulta de herbario y a los miembros de la Asociación Contisuyo, Bruce Owen, Antonio Oquiche y Karen Wise, por la contribución económica y logística.

\section{Literatura citada}

Arakaki, M. 1999. Flora vascular de la cuenca del Río Ilo-Moquegua, Perú. Tesis para optar el título profesional de Biólogo con mención en Botánica. Facultad de Ciencias Biológicas, Univ. Nac. Mayor de San Marcos.

Arakaki, M. y A. Cano. 2001. Vegetación y estado de conservación de la cuenca del Río IloMoquegua, Lomas de Ilo y áreas adyacentes. Arnaldoa 8(1): 49-70.

Brako, L. \& J. Zarucchi. 1993. Catalogue of the Flowering Plants and Gymnosperms of Peru. Missouri Botanical Garden. St. Louis.

Ferreyra, R. 1961. Las lomas costaneras del extremo sur del Perú. Boletín de la Sociedad Argentina de Botánica, 9: 85-120.

Ferreyra, R. 1983. Los tipos de vegetación de la costa peruana. Anales del Jardín Botánico de Madrid, 40:241-256.

Gentry, A. H. 1982. Neotropical floristic diversity: phytogeographical connections between Central and South America, Pleistocene climatic fluctuations, or an accident of the Andean orogeny? Ann. Missouri Botanical Garden. 69(3): 557-593.

Greig-Smith, P. 1964. Quantitative plant ecology. Butterworths. Washington.

INRENA (Instituto Nacional de Recursos Naturales). 1995. Estrategia del Sistema Nacional de Áreas Naturales Protegidas del Perú. Plan Director. Proyecto Fanpe.

Kalin, M., C. Villagrán, C. Marticorena y J. Armesto. 1982. Flora y relaciones biogeográficas en los Andes del norte de Chile $\left(18^{\circ}-19^{\circ} \mathrm{S}\right)$. En: Veloso A. \& Bustos, E. (eds.): El ambiente natural y las poblaciones humanas de los Andes del Norte Grande de Chile (Arica, Lat. $18^{\circ} 28^{\prime}$ S). Vol. 1. Rostlac, Montevideo: 71-92.

León, B., A. Cano y K. Young. 2003. Los helechos de las lomas costeras del Perú. Arnaldoa 9 (2): 7-42.

Rivas-Martínez, S. y O. Tovar. 1983. Síntesis biogeográfica de los Andes. Collectanea Botánica. Vol. 14: 515-521. Barcelona. 
Rodríguez, L. (ed.). 1996. Diversidad Biológica del Perú, zonas prioritarias para su conservación. Proyecto Fanpe GTZ-INRENA. Ministerio de Agricultura, Instituto Nacional de Recursos Naturales (INRENA), Lima.

Rundel, P., M. Dillon, M. Palma, \& S. Gulman. 1991. Phytogeography and ecology of the coastal Atacama and Peruvian deserts. Aliso 13: 1480

Weberbauer, A. 1945. El mundo vegetal de los Andes peruanos. Estudio Fitogeográfico. Estación Experimental Agrícola de La Molina. Dirección de Agricultura. Lima.
Young, K. y B. León. 1993. Distribución geográfica y conservación de las plantas acuáticas vasculares del Perú. En: Kahn, F., B. León y K. Young (comp.) Las plantas vasculares en las aguas continentales del Perú. Instituto Francés de Estudios Andinos. Lima.

Young, K. y A. Cano. 1994. Aporte florístico de la puna del Parque Nacional del Manu, Perú. Boletín de Lima. Vol. XVI, N. ${ }^{\circ}$ 91-96, pp. 381-393. Lima.

\section{Apéndice}

Lista de especies registradas para la Cuenca del Río llo-Moquegua y Lomas de llo, indicando formas de crecimiento $(\mathrm{H}=$ hierba, $\mathrm{S}=$ arbusto o sufrútice, $\mathrm{HS}=$ Hierba o arbusto, $\mathrm{T}=$ árbol, $\mathrm{P}$ = parásita) y especies endémicas ( ${ }^{*}$ del Perú, ${ }^{* *}$ endémicas para el Dpto. de Moquegua).

Taxa registrados

\section{Cuenca río Lomas Forma de Ilo-Moquegua Ilo crecimiento}

\begin{tabular}{|c|c|c|c|}
\hline DIVISION PTERIDOPHYTA & & & \\
\hline FAMILIA EQUISETACEAE & & & \\
\hline Equisetum bogotense Kunth & $\mathbf{X}$ & & $\mathrm{H}$ \\
\hline FAMILIA DRYOPTERIDACEAE & & & \\
\hline Cystopteris fragilis (L.) Bernh. & $X$ & & $\mathrm{H}$ \\
\hline FAMILIA PTERIDACEAE & & & \\
\hline Adiantum subvolubile Kuhn & $\mathrm{X}$ & & $\mathrm{H}$ \\
\hline Cheilanthes peruviana (Desv.) Moore & $\mathbf{X}$ & & $\mathrm{H}$ \\
\hline Cheilanthes pruinata Kaulf. & $\mathbf{X}$ & & $\mathrm{H}$ \\
\hline Pityrogramma trifoliata (L.) Tryon & & $X$ & $\mathrm{H}$ \\
\hline FAMILIA SALVINIACEAE & & & \\
\hline Azolla mexicana Presl. & $\mathbf{X}$ & & $\mathrm{H}$ \\
\hline DIVISION GNETOPHYTA & & & \\
\hline FAMILIA EPHEDRACEAE & & & \\
\hline Ephedra americana Humboldt \& Bonpland ex Willd. & $X$ & & $\mathrm{~S}$ \\
\hline Ephedra rupestris Bentham & $\mathbf{X}$ & & $\mathrm{S}$ \\
\hline DIVISION MAGNOLIOPHYTA & & & \\
\hline FAMILIA ACANTHACEAE & & & \\
\hline Dicliptera ruiziana Wasshausen & $\mathbf{X}$ & $X$ & $\mathrm{H}$ \\
\hline FAMILIA AIZOACEAE & & & \\
\hline Sesuvium portulacastrum (L.) L. & & $\mathbf{X}$ & $\mathrm{H}$ \\
\hline Tetragonia crystallina L' Héritier & $\mathbf{X}$ & & $\mathrm{H}$ \\
\hline Tetragonia ovata Phil. & $\mathbf{X}$ & $\mathbf{X}$ & $\mathrm{H}$ \\
\hline Tetragonia pedunculata Phil. & $\mathrm{X}$ & $X$ & $\mathrm{H}$ \\
\hline Tetragonia vestita I. M. Johnston & & 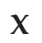 & $\mathrm{H}$ \\
\hline FAMILIA AMARANTHACEAE & & & \\
\hline Alternanthera halimifolia (Lamarck) Standley ex Pittier & $\mathrm{X}$ & & $\mathrm{H}$ \\
\hline Alternanthera sp. & $\mathbf{X}$ & & $\mathrm{H}$ \\
\hline Amaranthus dubius C. Martius ex Thellung & $\mathrm{X}$ & & $\mathrm{H}$ \\
\hline Amaranthus hybridus L. & $X$ & $\mathbf{X}$ & $\mathrm{H}$ \\
\hline Amaranthus viridis L. & $X$ & & $\mathrm{H}$ \\
\hline
\end{tabular}




\section{Cuenca río Lomas Forma de Ilo-Moquegua Ilo crecimiento}

Schinus molle L.

$\mathbf{X}$

$\mathrm{T}$

\section{FAMILIA APIACEAE}

Ammi visnaga (L.) Lamarck

Arracacia peruviana (H. Wolff) Constance *

Azorella compacta Philippi

Ciclospermum laciniatum (DC.) Constance

Ciclospermum leptophyllum (Persoon) Eichler

Domeykoa saniculifolia Mathias \& Constance*

Hydrocotyle bonariensis Comm. ex Lam.

FAMILIA ASCLEPIADACEAE

Asclepias physocarpa (E. Meyer) Schlechter

Sarcostemma andinum (Ball) R. Holm

FAMILIA ASTERACEAE

Acanthoxanthium spinosum (L.) Fourreau

Achyrocline alata (H.B.K.) DC.

Ambrosia arborescens Miller

Ambrosia artemisioides Meyen \& Walpers

Ambrosia peruviana Willdenow

Baccharis petiolata DC.

Baccharis tricuneata var. tricuneata

Baccharis sp.

Bidens exigua Sherff

Bidens pilosa L. var. pilosa

Centaurea melitensis L.

Chersodoma jodopappa (Schultz-Bip) Cabrera

Cichorium intybus L.

Chuquiraga rotundifolia Weddell

Conyza bonariensis (L.) Cronquist

Cotula australis (Sieber ex Sprengel) Hooker $\mathrm{f}$.

Diplostephium meyenii (Schultz-Bip) S. F. Blake

Diplostephium tacorense Hieronymus

Eupatorium sp.

Flaveria bidentis (L.) Kuntze

Galinsoga parviflora Cavanilles

Gamochaeta americana (Mill.) Weddell

Gamochaeta purpurea (L.) Cabrera

Gamochaeta sp.

Grindelia bergii (Hieronymus) Cabrera

Grindelia glutinosa (Cavanilles) Dunal

Grindelia tarapacana Philippi

Gynoxis longistyla (Greenman \& Cuatrecasas) Cuatrecasas **

Heliopsis sp. 1

Heliopsis sp. 2

Helogyne apaloidea Nuttall

Heterosperma ferreyrii $\mathrm{H}$. Robinson *

Heterosperma involucratum (Phil.) Reiche

Hypochaeris sp.

Mutisia acuminata var. hirsuta (Meyen) Cabrera

Onoseris minima Domke *

Ophryosporus heptanthus (Schultz-Bip. ex Wedd.) King \& Rob.

Ophryosporus peruvianus (Gmelin) King \& Robinson

Parastrephia lepidophylla (Wedd.) Cabrera

$\mathrm{X}$

\section{$X$}

$X$

$\mathrm{H}$

$\mathrm{H}$

$\mathbf{X}$

X

$\mathbf{X}$

X

$\mathbf{X}$

$X$

$\mathrm{x}$

X

$X$

$X$

X

X

X

$\mathrm{X}$

$X$

X

$X$

$X$

$\mathrm{x}$

$\mathrm{x}$

$\mathrm{X}$

$X$

$X$

$X$

$X$

$X$

X

$X$

$X$

$\mathrm{x}$

$\mathrm{x}$

$\mathrm{x}$

$\mathrm{X}$

$X$

$\mathrm{X}$

$X$

X

X

$X$

X

$X$

$\mathrm{X}$

$\mathrm{X}$

$\mathrm{H}$
$\mathrm{H}$

HS

S

S

S

S

S

$\mathrm{H}$

X H

X H

$S$

H

S

X H

$S$

S

S

X H

$\mathrm{H}$

X H

X H

$\mathrm{H}$

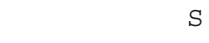

X S

S

S

S

S

HS

$\mathrm{H}$

$\mathrm{H}$

$\mathrm{H}$

S

$\mathrm{H}$

S

HS

$S$

(continúa...) 


\section{Taxa registrados}

\section{Cuenca río Lomas Forma de \\ Ilo-Moquegua Ilo crecimiento}

Picrosia longifolia D. Don

Polyachyrus annuus I. M. Johnston

Polyachyrus fuscus (Meyen) Walpers

Polyachyrus sphaerocephalus D. Don

Proustia berberidifolia (Cabr.) Ferreyra

Schkuhria pinnata (Lamarck) Kuntze var. pinnata

Senecio boliviensis Schultz-Bip

Senecio cumingii Hooker \& Arnott

Senecio neoviscosus Cuatrecasas **

Senecio phylloleptus Cuatrecasas *

Senecio rudbeckiaefolius Meyen \& Walpers *

Sonchus oleraceus L.

Stevia weberbaueri B. Robinson *

Tagetes multiflora H.B.K.

Taraxacum officinale Wiggers

Tessaria integrifolia R. \& P.

Trixis cacalioides H.B.K.

Vasquezia oppositifolia (Lagasca) S. F. Blake

Viguiera procumbens (Persoon) S. F. Blake

Werneria pygmophylla S. F. Blake **

Zinnia peruviana (L.) L.

FAMILIA BIGNONIACEAE

Tecoma arequipensis (Sprague) Sandwith

Tecoma fulva (Cavanilles) G. Don

$\begin{array}{lll} & \mathbf{X} & \mathrm{H} \\ & \mathbf{X} & \mathrm{H} \\ \mathbf{X} & \mathbf{X} & \mathrm{H} \\ \mathbf{X} & & \mathrm{H} \\ \mathbf{X} & & \mathrm{S} \\ \mathbf{X} & & \mathrm{H} \\ \mathbf{X} & & \mathrm{H} \\ \mathbf{X} & & \mathrm{H} \\ \mathbf{X} & & \mathrm{H} \\ \mathbf{X} & & \mathrm{S} \\ \mathbf{X} & & \mathrm{H} \\ \mathbf{X} & & \mathrm{H} \\ \mathbf{X} & & \mathrm{H} \\ \mathbf{X} & & \mathrm{H} \\ \mathbf{X} & & \mathrm{H} \\ \mathbf{X} & & \mathrm{S} \\ \mathbf{X} & \mathbf{X} & \mathrm{S} \\ \mathbf{X} & & \mathrm{H} \\ \mathbf{X} & & \mathrm{S} \\ \mathbf{X} & \mathbf{X} & \mathrm{H} \\ & & \mathrm{H} \\ \mathbf{X} & & \mathrm{S} \\ \mathbf{X} & & \\ & & \end{array}$

FAMILIA BORAGINACEAE

Amsinckia hispida (R. \& P.) I. M. Johnston

Cordia lutea Lamarck

Cryptantha parviflora (Philippi) Reiche

Cryptantha peruviana I. M. Johnston

Cryptantha sp.

Heliotropium arborescens L.

Heliotropium curassavicum L.

Heliotropium krauseanum Fedde

Heliotropium microstachyum R. \& P.

Heliotropium toratense I. M. Johnston **

Heliotropium sp.

Tiquilia litoralis (Philippi) A. Richardson

Tiquilia paronychioides (Philippi) A. Richardson

Tiquilia sp. 1

Tiquilia sp. 2

FAMILIA BRASSICACEAE

Brassica rapa subsp. campestris (L.) Clapham

Brayopsis calycina (Desvaux) Gilg \& Muschler

Capsella bursa-pastoris (L.) Medicus

Lepidium meyenii Walpers

Lepidium virginicum $\mathrm{L}$.

Lepidium sp. 1

Lepidium sp. 2

Mancoa hispida Weddell

Matthiola incana (L.) R. Brown

Raphanus satious L.

Rorippa nasturtium-aquaticum (L.) Hayek

$\mathrm{X}$

$\mathrm{X}$

$\mathrm{X}$

$\mathrm{X}$

$\mathrm{X}$

$\mathrm{x}$

$\mathrm{X}$

$\mathrm{X}$

$\mathrm{x}$

$\mathrm{x}$

$\mathrm{X}$

$\mathrm{X}$

$\mathrm{X}$

$\mathrm{X}$

$\mathrm{X}$

$\mathrm{X}$

$\mathrm{X}$

$\mathrm{x}$

$\mathrm{X}$

$X$

$\mathrm{X}$

$\mathrm{X}$

$\mathrm{X}$

$\mathrm{X}$
$\mathrm{H}$

$\mathrm{H}$

$\mathrm{H}$

$\mathrm{H}$

$S$

$\mathrm{H}$

$\mathrm{H}$

$\mathrm{H}$

$\mathrm{H}$

$S$

HS

$\mathrm{H}$

H S

$\mathrm{H}$

$\mathrm{H}$

S

$\mathrm{S}$

$\mathrm{H}$

$\mathrm{H}$

S

S

X H

S

$\begin{array}{ll}\text { X } & \text { H }\end{array}$

X $\mathrm{H}$

$\mathrm{H}$
$\mathrm{HS}$

HS

X H

X H

$\mathrm{H}$

HS

$\mathrm{S}$

X H

$\mathrm{H}$

$\mathrm{H}$

S

$\mathrm{H}$

$\mathrm{H}$

$\mathrm{H}$

$\mathrm{H}$

$\mathrm{H}$

$\mathrm{H}$

$\mathrm{H}$

$\mathrm{H}$

$\mathrm{H}$

$\mathrm{H}$

$\mathrm{H}$

(continúa...) 


\section{Cuenca río Lomas Forma de Ilo-Moquegua Ilo crecimiento}

Sisymbrium officinale (L.) Scopoli

Sisymbrium peruvianum DC.

Sisymbrium weberbaueri O. E. Schultz **

Weberbauera spathulaefolia (A. Gray) O. E. Schultz

\section{FAMILIA BROMELIACEAE}

Tillandsia purpurea R. \& P.

Tillandsia recurvata (L.) L.

Tillandsia sp.

\section{FAMILIA CACTACEAE}

Armatocereus sp.

Browningia candelaris (Meyen) Britton \& Rose

Corryocactus brevistylus (Schumann ex Vaupel) Britton \& Rose

Echinopsis pachanoi (Britton \& Rose) Friedrich \& G. Rowley

Echinopsis pampana (Britton \& Rose) D. R. Hunt

Echinopsis sp.

Haageocereus sp.

Neoraimondia arequipensis (Meyen) Backeberg *

Neowerdermannia sp.

Opuntia ignescens Vaupel

Opuntia sphaerica Foerster

Opuntia subulata (Muehlenpfordt) Engelmann

Oreocereus leucotrichus (Philippi) Wagenknecht

Weberbauerocereus torataensis F. Ritter **

FAMILIA CARYOPHYLLACEAE

Cerastium nanum Muschler *

Drymaria paposana Philippi var. serrulata Duke *

Drymaria paposana Philippi var. weberbaueri(Muschler) Duke *

Drymaria rotundifolia A. Gray

Pycnophyllum molle Remy

Spergularia collina I. M. Johnston *

Spergularia congestifolia I. M. Johnston *

Spergularia fasiculata Philippi

Spergularia sp.

FAMILIA CHENOPODIACEAE

Atriplex aff. herzogii Standl.

Chenopodium album $\mathrm{L}$.

Chenopodium ambrosioides L.

Chenopodium murale L.

Chenopodium petiolare H.B.K.

Chenopodium sp.

FAMILIA CONVOLVULACEAE

Convolvulus crenatifolius R. \& P.

Ipomoea sp.

Merremia dissecta (Jacquin) Hallier $\mathrm{f}$.

FAMILIA CRASSULACEAE

Crassula connata (R. \& P.) Berger

FAMILIA CUCURBITACEAE

Apodanthera mandonii Cogniaux

Cyclanthera mathewsii Arnott ex A. Gray *

Sicyos baderoa Hooker \& Arnott

FAMILIA CYPERACEAE

Cyperus corymbosus Rottboel

$\begin{array}{lll}\mathbf{X} & & \mathrm{H} \\ \mathbf{X} & & \mathrm{H} \\ \mathbf{X} & & \mathrm{H} \\ \mathbf{X} & & \mathrm{H} \\ & & \\ \mathbf{X} & \mathbf{X} & \mathrm{H} \\ \mathbf{X} & \mathbf{X} & \mathrm{H} \\ & \mathbf{X} & \mathrm{H}\end{array}$

$\mathrm{X}$

$\mathrm{X}$

$\checkmark$

$\mathrm{X}$

$\mathrm{X}$

$\mathrm{X}$

$\mathrm{X}$

$\mathrm{X}$

$\mathrm{X}$

$\mathrm{X}$

$\mathrm{X}$

X

$\mathrm{X}$

X

$\mathrm{x}$

X

X

$\mathrm{H}$ $\mathrm{H}$

S

S

S

S

$S$

S

S

$S$

$S$

$S$

$S$

S

S

S

H

$\mathrm{H}$

H

H

$\mathrm{H}$

$\mathrm{H}$

$\mathrm{H}$

$\mathrm{H}$

H

X

$\mathrm{H}$

H

$\mathrm{H}$

$\mathrm{H}$

HS

$\mathrm{H}$

H

HS

$\mathrm{H}$

X

$\mathrm{H}$

X

H

$\mathrm{X}$

X

H

H

X

(continúa...) 
Taxa registrados

\section{Cuenca río Lomas Forma de \\ Ilo-Moquegua Ilo crecimiento}

Cyperus tacnensis Nees \& Meyen

Eleocharis geniculata (L.) Roemer \& Schultes

Scirpus pungens M. Vahl

FAMILIA EUPHORBIACEAE

Chamaesyce hirta (L.) Millspaugh

Chamaesyce hypericifolia (L.) Millspaugh

Chamaesyce serpens (H.B.K.) Small

Croton alnifolius Lamarck *

Croton ruizianus Muell. Arg.

Croton sp.

Euphorbia peplus L.

Euphorbia sp.

Ricinus communis $\mathrm{L}$.

FAMILIA FABACEAE

Acacia macracantha Humboldt \& Bonpland ex Willdenow Adesmia miraflorensis Remy

Adesmia spinosissima Meyen ex J. Vogel

Astragalus peruvianus J. Vogel

Astragalus triflorus (DC.) Gray

Caesalpinia spinosa (Molina) Kuntze *

Crotalaria incana L. var. incana

Dalea moquehuana J. F. Macbride*

Dalea onobrychis DC.

Desmanthus virgatus (L.) Willdenow

Desmodium scorpiurus (Swartz) Desvaux

Hoffmannseggia miranda Sandwith *

Hoffmannseggia prostrata Lagergeim ex DC.

Hoffmannseggia stipulata Sandwith *

Hoffmannseggia ternata F. Philippi

Leucaena leucocephala (Lamarck) De Wit

Lupinus ballianus C. P. Smith

Lupinus toratensis C. P. Smith *

Lupinus sp. 1

Lupinus sp. 2

Medicago polymorpha L.

Medicago sativa L.

Melilotus indica (L.) Allioni

Otholobium pubescens (Poiret) Grimes

Prosopis sp.

Senna birostris (Domb. ex Vog.) H. Irwin \& Barn.

var. arequipensis (Vog.) H. Irwin \& Barn.

Vigna luteola (Jacquin) Bentham

FAMILIA GERANIACEAE

Balbisia meyeniana (Klotzsch) Steudel

Erodium cicutarium (L.) L' Héritier ex Aiton

Erodium malacoides (L.) L' Héritier

Geranium herrerae Knuth *

Geranium patagonicum Hook

Geranium sessiliflorum Cavanilles

FAMILIA HYDROPHYLLACEAE

Nama dichotomum (R. \& P.) Choisy

Phacelia pinnatifida Grisebach ex Weddell
$\mathrm{H}$

$\mathrm{X}$

$\mathrm{X}$

$\mathbf{X}$

$\mathrm{H}$

$\mathrm{X}$

$\mathrm{H}$

$\mathrm{X}$

$\mathrm{X}$

$\begin{array}{ll}X & \mathrm{H} \\ \mathbf{X} & \mathrm{H} \\ \mathrm{H}\end{array}$

$X$

$\mathrm{X}$

$X$

$\mathrm{X}$

$\mathrm{X}$

$\mathrm{X}$

$\mathrm{H}$

$\mathrm{H}$

$\mathrm{H}$

$\mathrm{H}$

S

$S$

$\mathrm{H}$

$\mathrm{H}$

$\mathrm{H}$

$\mathrm{H}$

$\mathrm{X}$

$\mathrm{T}$

$S$

$S$

$\mathrm{H}$

$\mathrm{H}$

$\mathrm{X}$

$\mathrm{X}$

X

$\mathrm{S}$

$\mathrm{H}$

$\mathrm{H}$

HS

HS

$\mathrm{H}$

$\mathrm{H}$

$\mathrm{X}$

$\mathrm{X}$

$\mathrm{H}$

$\mathrm{H}$

$\mathrm{S}$

S

$\mathrm{H}$

H S

$\mathrm{H}$

$\mathrm{H}$

$\mathrm{H}$

$\mathrm{H}$

$\mathrm{H}$

$\mathrm{T}$

$S$

$\mathrm{X}$

S

X H

X $\mathrm{X}$

X S

X H

X H

X H

X H

X H

X H

X H

(continúa...) 


\section{Cuenca río Lomas Forma de Ilo-Moquegua Ilo crecimiento}

\section{FAMILIA IRIDACEAE}

Olsynium junceum (E. Meyer ex J. S. Presl) Goldblatt

FAMILIA JUNCACEAE

Juncus bufonius L.

Juncus pallescens Lamarck

FAMILIA LAMIACEAE

Marrubium vulgare $\mathrm{L}$.

Salvia haenkei Bentham

Salvia rhombifolia R. \& P.

Satureja boliviana (Bentham) Briquet

FAMILIA LILIACEAE

Alstroemeria violacea Philippi

Asparagus officinalis L.

Bomarea ovata (Cavanilles) Mirbel

Nothoscordum bivalve (L.) Britton

Zephyranthes albicans (Herbert) Baker

Zephyranthes briquetii J. F. Macbride **

FAMILIA LOASACEAE

Cajophora carduifolia C. Presl. *

Caiophora superba Philippi

Mentzelia chilensis Gay

Nasa urens (Jacq.) Weigend

FAMILIA LOGANIACEAE

Buddleja coriacea Remy

FAMILIA LORANTHACEAE

Tristerix longebracteatus (Desrousseaux) Barlow \& Wiens

FAMILIA MALESHERBIACEAE

Malesherbia ardens J. F. Macbride **

Malesherbia arequipensis Ricardi *

FAMILIA MALVACEAE

Anoda cristata Schldl.

Cristaria multifida Cavanilles

Cristaria sp. 1

Cristaria sp. 2

Gossypium barbadense L.

Malva parviflora $\mathrm{L}$.

Malvastrum coromandelianum (L.) Garcke

Modiola caroliniana (L.) G. Don

Nototriche foetida Ulbrich *

Nototriche obcuneata (Baker f.) A. W. Hill

Nototriche pygmaea (Remy) Hill

Nototriche rugosa (Phil.) Hill

Palaua dissecta Bentham

Palaua weberbaueri Ulbrich

Sida cordifolia L.

Sida rhombifolia L.

Sida spinosa L.

Tarasa capitata (Cavanilles) Bates

Tarasa operculata (Cav.) Krapov.

Urocarpidium albiflorum Ulbrich *

Urocarpidium chilensis (Braun \& Bouché) Krapov.

FAMILIA MELIACEAE
$\mathbf{X}$

\section{$\mathrm{H}$}

$X$

$\mathrm{X}$

$\mathbf{X}$

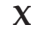

$\mathbf{X}$

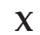

$\mathbf{X}$

$\mathrm{X}$

$\mathrm{X}$

$x$

$\mathbf{X}$

X

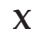

$\mathbf{X}$

$\mathbf{X}$

$X$

$\mathbf{X}$

$\mathbf{X}$ HS

X

$\mathrm{X}$

$\mathrm{X}$

$\mathrm{X}$

$\mathrm{X}$

$\mathrm{X}$

X

$\mathrm{X}$

$X$
$X$

$\mathrm{X}$

$\mathrm{X}$

$\mathrm{X}$

$\mathrm{X}$

$\mathrm{X}$

$X$

X
$\mathrm{H}$

$\mathrm{H}$

H

S

$\mathrm{H}$

S

H

$\mathrm{H}$

$\mathrm{H}$

H

$\mathrm{H}$

$\mathrm{H}$

$\mathrm{H}$

HS

$\mathrm{H}$

S

P

S

HS

H

$\mathrm{H}$

.

.

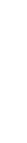

$\mathrm{H}$
$\mathrm{H}$
$\mathrm{H}$
$\mathrm{H}$
$\mathrm{H}$
$\mathrm{H}$


Taxa registrados

\section{Cuenca río Lomas Forma de \\ Ilo-Moquegua Ilo crecimiento}

Melia azedarach $\mathrm{L}$.

FAMILIA NYCTAGINACEAE

Boerhavia diffusa L.

Boerhavia erecta L.

Boerhavia sp.

Bouganvillea spinosa (Cavanilles) Heimerl

Colignonia parviflora var. biumbellata (Ball) Bohlin*

Mirabilis prostrata (R. \& P.) Heimerl

Mirabilis sp.

FAMILIA ONAGRACEAE

Epilobium denticulatum R. \& P.

Oenothera arequipensis Munz \& I. M. Johnson

Oenothera nana Grisebach

Oenothera rosea Aiton

Oenothera versicolor Lehmann

FAMILIA OXALIDACEAE

Oxalis sp.

FAMILIA PAPAVERACEAE

Argemone mexicana L. var. mexicana

Argemone subfusiformis Ownbey subsp. subfusiformis

FAMILIA PASSIFLORACEAE

Passiflora foetida L.

Passiflora peduncularis Cavanilles *

Passiflora sp.

FAMILIA PLANTAGINACEAE

Plantago limensis Pers. *

Plantago major L.

FAMILIA POACEAE

Anthochloa lepidula Nees \& Meyen

Aristida adscensionis L.

Aristida sp.

Bothriochloa sp.

Bromus catharticus M. Vahl var. catharticus

Calamagrostis preslii (Kunth) A. Hitchcock

Calamagrostis violacea (Weddell) A. Hitchcock

Cenchrus echinatus L.

Cenchrus incertus M. Curtis *

Cenchrus myosuroides H.B.K.

Chloris radiata (L.) Swartz

Chloris virgata Swartz

Cortaderia jubata (Lemaire) Stapf.

Cynodon dactylon (L.) Persoon

Cynodon maritimus H.B.K.

Distichlis spicata (L.) Greene

Echinochloa crus-pavonis (H.B.K.) Schultes

Enneapogon desvauxii P. Beauvois

Eragrostis cilianensis (Allioni) Vignolo-Lutati ex Janchen

Eragrostis mexicana (Hornemann) Link subsp. virescens

Eragrostis nigricans (H.B.K.) Steudel

Eragrostis peruviana (Jacquin) Trinius

Eragrostis sp.

Eriochloa polystachya H.B.K.
X

T

$\mathrm{X}$

$\mathrm{X}$

$\mathrm{X}$

$\mathrm{X}$

$\mathrm{X}$

$\mathrm{x}$

$\mathrm{x}$

$\mathrm{X}$

$\mathrm{X}$

$\mathrm{x}$

$\mathrm{X}$

$\mathbf{X}$

$\mathrm{x}$

$\mathrm{X}$

$\mathrm{x}$

$\mathrm{X}$

$\mathrm{x}$

X T

S

$\mathrm{H}$

$\mathrm{H}$

$\mathrm{S}$

$\mathrm{H}$

$\mathrm{H}$

$\mathrm{H}$

$\mathrm{H}$

$\mathrm{H}$

$\mathrm{H}$

$\mathrm{H}$

$\mathrm{H}$

$\mathrm{H}$

X

$\mathrm{H}$

$\mathrm{H}$

$\mathrm{H}$

$\mathrm{H}$

$\mathrm{H}$

X

$\mathrm{H}$

$\mathrm{H}$

$\mathrm{X}$

$\mathrm{X}$

$\mathrm{X}$

$\mathrm{X}$

$\mathrm{X}$

$\mathrm{X}$

$\mathrm{X}$

$x$

$\mathrm{X}$
$\mathrm{X}$

$X$
$X$

X

X

$\mathrm{X}$

X

$\mathrm{X}$

$\mathrm{X}$

$\mathrm{X}$

$\mathrm{X}$

$\mathrm{X}$

X

X

$\mathrm{H}$

$\mathrm{H}$

$\mathrm{H}$

$\mathrm{H}$

H

H

$\mathrm{H}$

X

X

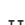

$\mathrm{H}$

$\mathrm{H}$

$\mathrm{H}$

$\mathrm{H}$

$\mathrm{H}$

X

$\mathrm{H}$

$\mathrm{H}$

$\mathrm{H}$

$\mathrm{H}$

$\mathrm{H}$

$\mathrm{H}$

H

$\mathrm{H}$

$\mathrm{H}$

(continúa...) 


\section{Cuenca río Lomas Forma de \\ Ilo-Moquegua Ilo crecimiento}

\begin{tabular}{|c|c|c|c|}
\hline Festuca dolichophylla J. S. Presl & $\mathrm{x}$ & & $\mathrm{H}$ \\
\hline Festuca orthophylla Pilger & $\mathrm{X}$ & & $\mathrm{H}$ \\
\hline Leptochloa uninervia (Presl.) Hitch. \& Chase & $\mathrm{X}$ & & $\mathrm{H}$ \\
\hline Muhlenbergia peruviana (P. Beauvois) Steudel & $\mathrm{X}$ & & $\mathrm{H}$ \\
\hline Nassella nardoides (Philippi) Barkworth & & $\mathrm{x}$ & $\mathrm{H}$ \\
\hline Nassella neesiana (Trinius \& Ruprecht) Barkworth & $\mathrm{x}$ & & $\mathrm{H}$ \\
\hline Nassella pubiflora (Trinius \& Ruprecht) Desvaux & $\mathbf{X}$ & & $\mathrm{H}$ \\
\hline Paspalum sp. & & $\mathrm{x}$ & s \\
\hline Phragmites australis (Cav.) Trinius ex Steudel & $\mathbf{X}$ & & $\mathrm{H}$ \\
\hline Polypogon elongatus H.B.K. & $\mathrm{x}$ & & H \\
\hline Polypogon interruptus H.В.К. & $\mathrm{x}$ & & $\mathrm{H}$ \\
\hline Polypogon monspeliensis (L.) Desfontaines & $\mathrm{x}$ & & $\mathrm{H}$ \\
\hline Setaria parviflora (Poiret) Kerguélen & $\mathrm{X}$ & & $\mathrm{H}$ \\
\hline Setaria verticillata (L.) Beauvois & $\mathrm{X}$ & & $\mathrm{H}$ \\
\hline Setaria sp. & $\mathrm{x}$ & & $\mathrm{H}$ \\
\hline Sorghum bicolor (L.) Moench & $\mathrm{X}$ & & $\mathrm{H}$ \\
\hline Stipa апnиа Mez & $\mathrm{X}$ & & $\mathrm{H}$ \\
\hline Stipa ichu (R.\& P.) Kunth & $\mathrm{X}$ & & $\mathrm{H}$ \\
\hline Tragus berteronianus Schultes & & $\mathrm{x}$ & $\mathrm{H}$ \\
\hline Tragus racemosus (L.) Allioni & & $\mathrm{x}$ & $\mathrm{H}$ \\
\hline Urochloa mutica (Forsk.) Nguyen & & $\mathrm{X}$ & $\mathrm{H}$ \\
\hline \multicolumn{4}{|l|}{ FAMILIA POLEMONIACEAE } \\
\hline Cantua buxifolia Juss. ex Lam. & $\mathrm{X}$ & & $\mathrm{s}$ \\
\hline Gilia laciniata R. \& P. var. laciniata & & $\mathrm{X}$ & $\mathrm{H}$ \\
\hline \multicolumn{4}{|l|}{ FAMILIA POLYGALACEAE } \\
\hline Monnina macrostachya R. \& P. * & $\mathrm{X}$ & & $\mathrm{s}$ \\
\hline \multicolumn{4}{|l|}{ FAMILIA POLYGONACEAE } \\
\hline Polygonum hydropiperoides Michaux & $\mathrm{x}$ & & $\mathrm{H}$ \\
\hline \multicolumn{4}{|l|}{ FAMILIA PORTULACACEAE } \\
\hline Portulaca oleracea $\mathrm{L}$. & $\mathrm{x}$ & & $\mathrm{H}$ \\
\hline Portulaca perennis R. E. Fries & $\mathrm{x}$ & & H \\
\hline \multicolumn{4}{|l|}{ FAMILIA PRIMULACEAE } \\
\hline Anagallis arvensis $\mathrm{L}$. & $\mathrm{X}$ & & $\mathrm{H}$ \\
\hline \multicolumn{4}{|l|}{ FAMILIA RANUNCULACEAE } \\
\hline Clematis millefoliata Eichler & $\mathbf{X}$ & & $\mathrm{s}$ \\
\hline \multicolumn{4}{|l|}{ FAMILIA RHAMNACEAE } \\
\hline Colletia spinosissima J. Gmelin & $\mathrm{X}$ & & $\mathrm{s}$ \\
\hline \multicolumn{4}{|l|}{ FAMILIA ROSACEAE } \\
\hline Kageneckia lanceolata R. \& P. & $\mathrm{x}$ & & $\mathrm{s}$ \\
\hline Margyricarpus sp. & $\mathrm{x}$ & & s \\
\hline Polylepis besseri Hieronymus & $\mathrm{X}$ & & $\mathrm{T}$ \\
\hline \multicolumn{4}{|l|}{ FAMILIA RUBIACEAE } \\
\hline Galium weberbaueri Krause * & $\mathrm{X}$ & & $\mathrm{H}$ \\
\hline \multicolumn{4}{|l|}{ FAMILIA SANTALACEAE } \\
\hline Quinchamalium lomae Pilger* & & $\mathbf{x}$ & $\mathrm{H}$ \\
\hline Quinchamalium procumbens R. \& P. & $\mathrm{X}$ & & $\mathrm{H}$ \\
\hline Quinchamalium sp. & $\mathrm{X}$ & & HS \\
\hline \multicolumn{4}{|l|}{ FAMILIA SAPINDACEAE } \\
\hline Cardiospermum corindum $\mathrm{L}$. & & $\mathbf{x}$ & $\mathrm{H}$ \\
\hline Sapindus saponaria L. & $\mathrm{X}$ & & $\mathrm{T}$ \\
\hline \multicolumn{4}{|l|}{ FAMILIA SCROPHULARIACEAE } \\
\hline Bartsia serrata Molau & $\mathrm{X}$ & & $\mathrm{H}$ \\
\hline
\end{tabular}




\section{Cuenca río Lomas Forma de \\ Ilo-Moquegua Ilo crecimiento}

Bartsia weberbaueri Diels

Calceolaria angustiflora R. \& P. *

Calceolaria inamoena Kraenzlin subsp. inamoena

Calceolaria lobata Cavanilles

Calceolaria parvifolia Weddell subsp. parvifolia

Calceolaria pisacomensis Meyen ex Walpers *

Calceolaria plectranthifolia Walpers

Calceolaria sclerophylla Molau **

Mimulus glabratus H.B.K.

Veronica anagallis-aquatica $\mathrm{L}$.

$\begin{array}{ll}\mathbf{X} & \mathrm{H} \\ \mathbf{X} & \mathrm{S} \\ \mathbf{X} & \mathrm{S} \\ \mathbf{X} & \mathrm{H} \\ \mathbf{X} & \mathrm{S} \\ \mathbf{X} & \mathrm{S} \\ \mathbf{X} & \mathrm{H} \\ \mathbf{X} & \mathrm{S} \\ \mathbf{X} & \mathrm{H} \\ \mathbf{X} & \mathrm{H}\end{array}$

\section{FAMILIA SOLANACEAE}

Datura stramonium L.

Dunalia spinosa (Meyen) Dammer

Exodeconus flavus (I. M. Johnston) Axelius \& D'Arcy

Fabiana stephanii Hunziker \& Barboza

Leptoglossis acutiloba (I. M. Johnston) Hunziker \& Subils * Leptoglossis albiflora (I. M. Johnston) Hunziker \& Subils * Lycopersicon chilense Dunal

Lycopersicon esculentum Miller var. esculentum

Lycopersicon peruvianum var. peruvianum (L.) Miller

Nicandra physaloides (L.) Gaertner

Nicotiana glauca Graham

Nicotiana paniculata L. *

Nolana arenicola Johnston *

Nolana gracillima (I. M. Johnston) I. M. Johnston *

Nolana johnstonii Vargas *

Nolana pallidula I. M. Johnston *

Nolana pilosa Johnston *

Nolana platyphylla (I. M. Johnston) I. M. Johnston **

Nolana sp.

Salpichroa weberbaueri (Dammer) J. F. Macbride

Solanum acroscopicum Ochoa *

Solanum americanum Miller

Solanum chrysotrichum Schlechtendal

Solanum corymbosum Jacquin

Solanum multifidum Lamarck

Solanum nitidum R. \& P.

Solanum phyllanthum Cavanilles

Solanum radicans $\mathrm{L}$. $\mathrm{f}$.

Solanum tacnaense Ochoa

Solanum sp. 1

Solanum sp. 2

FAMILIA STERCULIACEAE

Waltheria ovata Cavanilles

Waltheria sp. 1

Waltheria sp. 2

FAMILIA TROPAEOLACEAE

Tropaeolum majus L.

Tropaeolum tuberosum $\mathrm{L}$. 


\section{Taxa registrados}

\section{Cuenca río Lomas Forma de Ilo-Moquegua Ilo crecimiento}

Urtica urens $\mathrm{L}$.

$\mathrm{X}$

$\mathrm{H}$

FAMILIA VALERIANACEAE

Valeriana nivalis Weddell

Valeriana warburgii Graebner *

X H

FAMILIA VERBENACEAE

Junellia juniperina (Lagasca) Moldenke

Lantana scabiosaeflora H.B.K. var. scabiosaeflora

Lippia nodiflora (L.) Michaux

Lippia sp.

Pitraea cuneato-ovata (Cav.) Caro

Verbena clavata R. \& P. var. clavata

Verbena litoralis H.B.K. fo. litoralis

X H

FAMILIA VITACEAE

Vitis vinifera L.

X S

X S

X $\quad$ X

X H

$\begin{array}{lll}\mathbf{X} & \mathbf{X}\end{array}$

$\mathrm{X}$

X H

FAMILIA ZYGOPHYLLACEAE

Fagonia chilensis Hooker \& Arnott

Tribulus terrestris L.

$\mathbf{X}$

$\mathrm{H}$

X H

X H 